\title{
Validity of the diagnosis of pneumonia in hospitalised patients with COPD
}

\author{
Lydia J. Finney (1) 1 , Vijay Padmanaban², Samuel Todd², Nadia Ahmed ${ }^{1}$, \\ Sarah L. Elkin ${ }^{2,3}$ and Patrick Mallia (1) 1,2,3
}

Affiliations: ${ }^{1}$ National Heart and Lung Institute, Imperial College, London, UK. ${ }^{2}$ Imperial College Healthcare NHS Trust, London, UK. ${ }^{3}$ These authors contributed equally.

Correspondence: Patrick Mallia, National Heart and Lung Institute, Imperial College, Norfolk Place, London, W2 1PG, UK. E-mail: p.malliađimperial.ac.uk

\section{ABSTRACT}

Rationale: Exacerbations of chronic obstructive pulmonary disease (COPD) and pneumonia are two of the most common reasons for acute hospital admissions. Acute exacerbations and pneumonia present with similar symptoms in COPD patients, representing a diagnostic challenge with a significant impact on patient outcomes. The objectives of this study were to compare the prevalence of radiographic consolidation with the discharge diagnoses of hospitalised COPD patients.

Methods: COPD patients admitted to three UK hospitals over a 3-year period were identified. Participants were included if they were admitted with an acute respiratory illness, COPD was confirmed by spirometry and a chest radiograph was performed within $24 \mathrm{~h}$ of admission. Pneumonia was defined as consolidation on chest radiograph reviewed by two independent observers

Results: There were 941 admissions in 621 patients included in the final analysis. In 235 admissions, consolidation was present on chest radiography and there were 706 admissions without consolidation. Of the 235 admissions with consolidation, only $42.9 \%$ had a discharge diagnosis of pneumonia; $90.7 \%$ of patients without consolidation had a discharge diagnosis of COPD exacerbation. The presence of consolidation was associated with increased rate of high-dependency care admission, increased mortality and prolonged length of stay. Inhaled corticosteroid use was associated with recurrent pneumonia.

Conclusions: Pneumonia is underdiagnosed in patients with COPD. Radiographic consolidation is associated with worse outcomes and prolonged length of stay. Incorrect diagnosis could result in inappropriate use of inhaled corticosteroids. Future guidelines should specifically address the diagnosis and management of pneumonia in COPD.

\section{@ERSpublications}

Pneumonia is common in hospitalised COPD patients but is frequently not recognised and underdiagnosed. This has implications for the correct risk stratification and treatment of COPD patients. http://bit.ly/2HTfIKo

Cite this article as: Finney LJ, Padmanaban V, Todd S, et al. Validity of the diagnosis of pneumonia in hospitalised patients with COPD. ERJ Open Res 2019; 5: 00031-2019 [https://doi.org/10.1183/ 23120541.00031-2019]. 


\section{Introduction}

Chronic obstructive pulmonary disease (COPD) is the fourth leading cause of death worldwide [1]. It accounts for 30000 deaths every year in the UK alone and is the second most common reason for emergency hospital admission, resulting in 90000 admissions and 1 million bed-days per annum. Community-acquired pneumonia is also a common cause of hospital admission and death, and COPD patients are at increased risk of pneumonia [2,3]. Acute exacerbations and pneumonia present with similar symptoms including dyspnoea, cough, sputum production and fever. Diagnosis of pneumonia is based on the symptoms and signs of an acute lower respiratory tract infection confirmed by a chest radiograph showing new shadowing that is not due to any other cause [4]. The diagnosis of pneumonia on clinical grounds alone has poor sensitivity and specificity, and the presence of radiographic consolidation is usually required [5]. The diagnosis of pneumonia in hospital patients is often inaccurate [6, 7] but this has not been examined specifically in a COPD population.

The Global Initiative for Chronic Obstructive Lung Disease (GOLD) strategy documents states that diagnosis of a COPD exacerbation requires exclusion of other causes of respiratory symptoms, including pneumonia [8]. However, it has been consistently reported across a range of healthcare settings that a substantial proportion of patients with a primary diagnosis of COPD exacerbation have radiological evidence of pneumonia [9-11]. The prevalence of pneumonia in COPD exacerbations and its effect on outcomes has been difficult to determine due to differing diagnostic labels used in hospitalised COPD patients admitted with respiratory symptoms and radiographic consolidation.

We examined pneumonia and COPD exacerbations by identifying all COPD patients admitted to three hospitals with acute respiratory symptoms over a 3-year period. We collected data on their discharge diagnoses and independently assessed the presence of consolidation on chest radiograph to determine the frequency of radiographic consolidation, and how this relates to discharge diagnoses, clinical outcomes, inflammatory markers and microbiology.

\section{Methods}

All COPD patients admitted to three London teaching hospitals between January 1, 2010 and December 31, 2012 were identified. Inclusion criteria were a clinical diagnosis of COPD confirmed with spirometry (forced expiratory volume in $1 \mathrm{~s}(\mathrm{FEV} 1) /$ forced vital capacity ratio $(\mathrm{FVC})<0.7)$ performed when the patient was clinically stable, admission with an acute respiratory illness and a chest radiograph performed within $24 \mathrm{~h}$ of admission. All patients were either current or ex-smokers. The European Coal and Steel Community reference equations for calculating per cent predicted spirometry values were used. Patients in whom another cause for their admission was identified and immunosuppressed patients were excluded.

\section{Case definition of pneumonia}

Admission chest radiographs were reviewed by two independent observers blinded to the final discharge diagnosis. One of these was the radiologist performing routine reporting of chest radiographs that would have been available during the admission. The second observer was a member of the study team (L.J. Finney) who reviewed the chest radiograph retrospectively. In the case of disagreement between the two, a second respiratory physician (P. Mallia) independently reviewed the chest radiograph. Pneumonia was defined as consolidation on the chest radiograph compatible with a diagnosis of pneumonia in the opinion of two reviewers. An exacerbation was defined as an admission with no radiographic consolidation. Discharge diagnoses were obtained from the hospital electronic discharge system. Demographic and clinical data were obtained from the chronic disease management system and electronic hospital records. Recurrent pneumonia was defined as two or more admissions with consolidation during the study period and recurrent exacerbations were defined as two or more admissions with no consolidation during the study period.

\section{Analysis}

Data were analysed using statistical software package STATA version 14 IC (StataCorp, College Station, TX, USA). To avoid duplicate data, patient characteristics were assessed based on the radiological diagnosis for the subject's first admission. All admissions were then analysed according to final radiological diagnosis. Data are presented as mean \pm SD or median (interquartile range) for continuous variables and frequency (percentages) for categorical variables. Data were assessed for normality. Continuous variables were studied using a Student's t-test, Mann-Whitney U-test or Kruskal-Wallis test as appropriate. Categorical variables were assessed using Fishers exact test or $\chi^{2}$-test as appropriate. Multiple linear regression analysis was used to assess whether consolidation on chest radiograph was associated with greater disease severity independent of discharge diagnosis. C-reactive protein (CRP), white blood cell count and length of stay data were logarithmically transformed for this analysis. 


\section{Results}

\section{Patient demographics}

1011 hospital admissions were identified during the study period. 70 admissions were excluded (56 had another primary reason for admission, five were immunosuppressed patients and nine patients had no chest radiograph) and 941 admissions in 621 patients were included in the final analysis (figure 1). The median (interquartile range) age of the patients was 72 (65-79) years, 114 (18.3\%) were current smokers and mean \pm SD FEV1 was $43 \pm 18 \%$ predicted.

\section{Baseline characteristics by diagnosis on first admission}

Of these 621 patients, 437 (70.4\%) patients had no radiographic consolidation on their first admission and 184 (29.6\%) patients had consolidation on their first admission. Patients with consolidation were significantly older than those admitted with an exacerbation and were significantly more likely to have had a previous admission for pneumonia (table 1). Patients in whom consolidation was not present on their first admission had more frequent admissions throughout the study period. There were no significant differences between groups for severity of airflow obstruction, smoking status or body mass index. Ischaemic heart disease was the most common comorbidity, identified in $36.2 \%$ of patients. Diabetes was present in $16.9 \%$ of patients and bronchiectasis was present in $10.7 \%$ of patients. There was no difference in presence of comorbidities between groups (table 1). There was no difference in overall inhaled corticosteroid (ICS) use or beclomethasone-equivalent dose between the two groups. However, there was greater use of budesonide in patients with COPD exacerbation (table 1).

\section{Discrepancies between clinical and radiological diagnoses}

Following review of the admission chest radiographs, 235 admissions were identified with radiographic consolidation and 706 admissions without consolidation. In eight cases, there were discrepancies in the interpretation of the radiograph that were resolved by the second respiratory physician. Of the 235 admissions with confirmed consolidation, 101 (43\%) had a diagnosis of pneumonia recorded on their

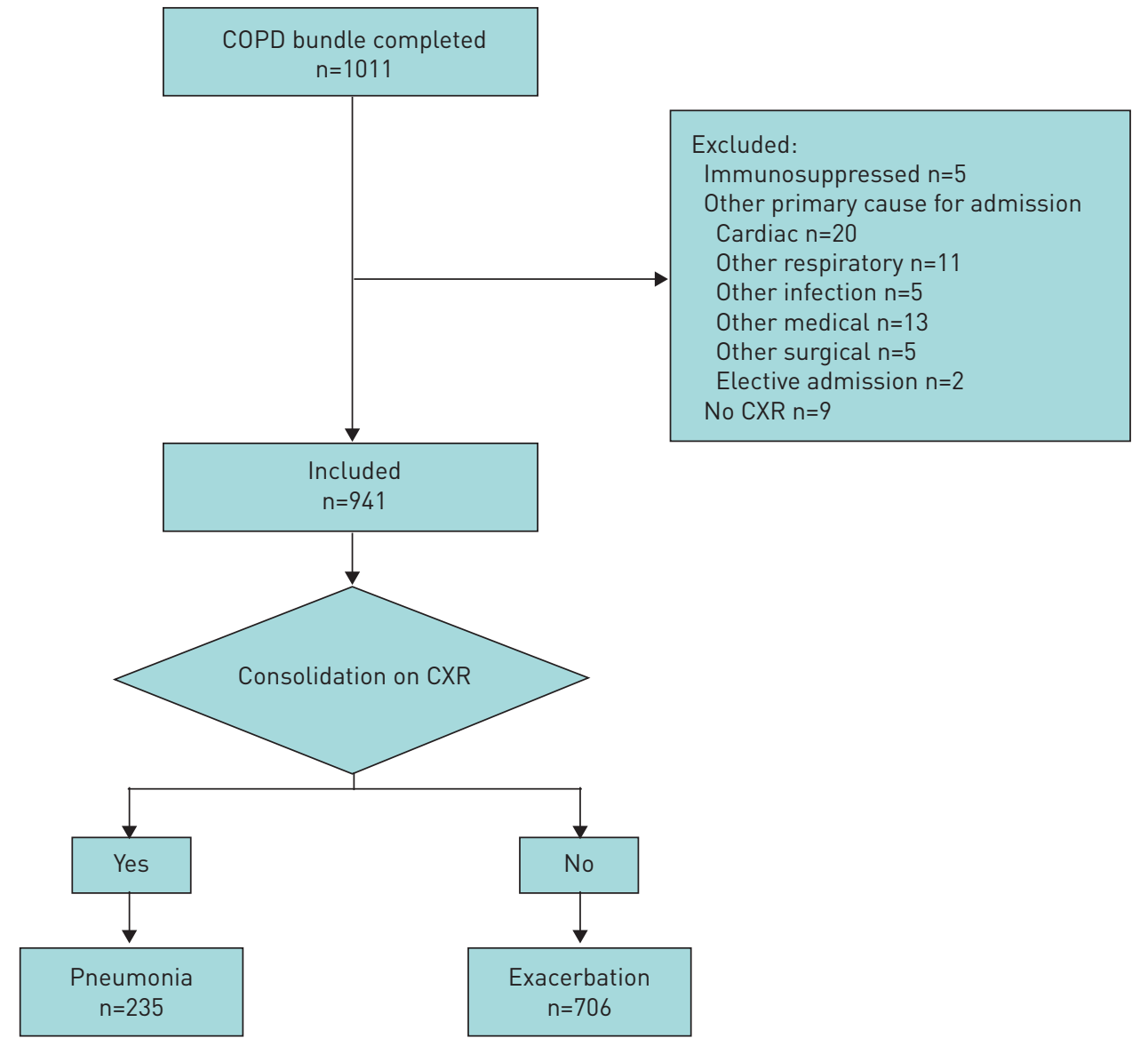

FIGURE 1 Flow diagram of patient episodes included in study. COPD: chronic obstructive pulmonary disease; CXR: chest radiograph. 
TABLE 1 Baseline characteristics and therapies of chronic obstructive pulmonary disease patients by radiological diagnosis on first admission

\begin{tabular}{|c|c|c|c|}
\hline & Exacerbation & Pneumonia & p-value \\
\hline Patients & 437 & 184 & \\
\hline Age years mean \pm sD & $70.61 \pm 10.70$ & $74.08 \pm 9.36$ & 0.0002 \\
\hline Males & $51 \%$ & $52 \%$ & 1.0 \\
\hline FEV $1 \%$ predicted & $41(21-56)$ & 39 (30-59) & 0.49 \\
\hline FEV1 L & $0.955(0.7-1.25)$ & $0.96(0.675-1.425)$ & 0.82 \\
\hline FEV $1 / F V C$ & $48 \%(38-57 \%)$ & $44 \%(35.5-57.5 \%)$ & 0.54 \\
\hline Body mass index $\mathrm{kg} \cdot \mathrm{m}^{-2}$ & $23(20-29)$ & $23(18.3-27.0)$ & 0.72 \\
\hline Current smoker & $83(18.4 \%)$ & $33(17.4 \%)$ & 1.0 \\
\hline Bronchiectasis & $45(10 \%)$ & $21(11.1 \%)$ & 0.69 \\
\hline Ischaemic heart disease & $154(35.2 \%)$ & $70(38.5 \%)$ & 0.46 \\
\hline Type II diabetes & $74(16.9 \%)$ & $31(17.0 \%)$ & 1.0 \\
\hline Previous stroke & $16(3.5 \%)$ & $4(2.1 \%)$ & 0.49 \\
\hline Admission for pneumonia in last year & $22(5 \%)$ & $31(16.8 \%)$ & $<0.001$ \\
\hline Total number of admissions in study period & $2.37 \pm 3.59$ & $1.48 \pm 3.01$ & 0.002 \\
\hline Inhaled corticosteroid users & $344(78.7 \%)$ & $138(75.8 \%)$ & 0.45 \\
\hline Fluticasone users & $236(52.4 \%)$ & $112(59.0 \%)$ & 0.19 \\
\hline Budesonide users & $97(22.0 \%)$ & $23(12.1 \%)$ & 0.01 \\
\hline Beclomethasone equivalent dose $\mathrm{mg} \cdot \mathrm{day}^{-1}$ & $1600(400-2000)$ & $1000(0-2000)$ & 0.87 \\
\hline Long-term oxygen therapy users & $61(13.9 \%)$ & $33(18.3 \%)$ & 0.18 \\
\hline
\end{tabular}

Data are presented as $\mathrm{n}(\%)$ or median (interquartile range) unless otherwise stated. $\mathrm{FEV} 1$ : forced expiratory volume in $1 \mathrm{~s}$; FVC: forced vital capacity.

discharge summary, 126 (53.6\%) patients had a diagnosis of COPD exacerbation and the remaining eight (3.4\%) had a diagnosis of lower respiratory tract infection or shortness of breath. In only nine admissions were both COPD exacerbation and pneumonia listed as discharge diagnoses, and in these cases, the primary diagnosis was used for our analysis. Of the 706 admissions with no radiological consolidation, 644 $(91.2 \%)$ had a discharge diagnosis of COPD exacerbation, $44(6.2 \%)$ had a diagnosis of pneumonia and diagnosis was lower respiratory tract infection or shortness of breath in 18 patients (2.6\%).

\section{Relationship between consolidation and outcomes and treatment}

The presence of radiographic consolidation was associated with a significantly longer length of stay, increased rates of admission to a high-dependency unit and increased in-hospital mortality compared to admissions with no consolidation (tables 2 and 3). Peripheral white cell count and serum CRP were higher in admissions with consolidation. Patients with a discharge diagnosis of exacerbation but who had radiographic consolidation were significantly more likely to be treated with oral corticosteroids compared to patients with both a discharge diagnosis of pneumonia and consolidation $(97.56 \%$ versus $56.25 \%, \mathrm{p}<0.01)$. Patients were prescribed antibiotics in $853(94.1 \%)$ admissions. The most frequently prescribed antibiotic for patients with a discharge diagnosis of exacerbation was doxycycline (47\%). The most commonly prescribed treatment for patients with a discharge diagnosis of pneumonia was amoxicillin and clarithromycin (28\%).

\section{Microbiology}

$18.7 \%$ of patients had a positive sputum culture on their first admission. The most commonly isolated organism was Pseudomonas aeruginosa, in 5\% of admissions. Haemophilus influenzae was isolated in 18

TABLE 2 Clinical markers of admission severity by hospital episode

\begin{tabular}{lccc} 
& Exacerbation & Pneumonia & p-value \\
\hline Length of hospital admission days & $6(3-10)$ & $9(5-15)$ & $<0.001$ \\
In-hospital mortality & $9(1.3 \%)$ & $11(4.6 \%)$ & $<0.001$ \\
High-dependency or intensive care admission & $99(14.0 \%)$ & $48(21.6 \%)$ & 0.0212 \\
C-reactive protein $\mathbf{n g} \cdot \mathbf{m L}^{-1}$ & $18.9(6.5-54)$ & $102.65(44.5-203)$ & $<0.001$ \\
White blood cell count cells $\mathbf{c m}^{-3}$ & $10.3(7.8-13.6)$ & $14.4(10.15-18.95)$ & $<0.001$ \\
Platelet count cells $\mathbf{c m}^{-3}$ mean \pm sd & $260.22 \pm 91.57$ & $254.43 \pm 99.42$ & 0.46 \\
\hline Data are presented as median (interquartile range) or $\mathrm{n}(\%)$ unless otherwise stated. &
\end{tabular}




\begin{tabular}{|c|c|c|}
\hline & Coefficient $(95 \% \mathrm{Cl})$ & p-value \\
\hline Log length of stay constant & 1.7135 (1.6454-1.7817) & \\
\hline Discharge diagnosis pneumonia & $0.2886(0.1084-0.4688)$ & 0.002 \\
\hline Consolidation on radiograph & $0.3533(0.2030-0.5037)$ & $<0.001$ \\
\hline Log C-reactive protein constant & $2.8524(2.7336-2.9712)$ & \\
\hline Discharge diagnosis pneumonia & $0.6776(0.3724-0.9828)$ & $<0.001$ \\
\hline Consolidation on radiograph & $1.199(0.9419-1.4578)$ & $<0.001$ \\
\hline Log white blood cell count constant & $2.3185(2.2862-2.3509)$ & \\
\hline Discharge diagnosis pneumonia & $0.1242(0.0384-0.2099)$ & 0.005 \\
\hline Consolidation on radiograph & $0.2410(0.1698-0.3122)$ & $<0.001$ \\
\hline
\end{tabular}

(2.8\%) admissions and Streptococcus pneumoniae in 13 (2.0\%). Other Gram-negative organisms were identified in $9 \%$ of admissions. There was no significant difference in the number of days since last admission between patients with a Gram-negative organisms (170.7 \pm 31.3 days since last admission) compared to those who did not have a Gram-negative organism (193.2 \pm 204 days). P. aeruginosa was the most commonly identified organism in 17 (10.4\%) of all pneumonia admissions and $34(8.65 \%)$ exacerbations. Other Gram-negative organisms were identified in $26(11.1 \%)$ pneumonias and $58(8.2 \%)$ exacerbations. There was no significant difference in organisms isolated between admissions for pneumonia and exacerbations (table 4).

\section{Multiple admissions}

There were 67 patients with multiple admissions with consolidation during the study period. Factors associated with multiple admissions were comorbid ischaemic heart disease (OR 1.90, 95\% CI 1.14-3.18), chronic kidney disease (OR 4.03, 95\% CI 1.57-10.24), increasing age (OR 1.04, 95\% CI 1.01-1.07) and treatment with ICSs (OR 2.10, 95\% CI 1.05-4.22). There was a trend towards increased risk of recurrent pneumonia if a Gram-negative organism was present in sputum on the first admission (OR 1.82, 95\% CI 0.98-3.40; $\mathrm{p}=0.059)$. Multivariate analysis showed chronic kidney disease, increasing age and treatment with ICSs were independently associated with increased risk of multiple admissions with consolidation. 104 patients had two or more admissions with no consolidation during the study period. Factors associated with an increased risk of two or more admissions were concomitant bronchiectasis (OR 2.56, 95\% CI 1.44-4.56), current smoking (OR 1.65, 95\% CI 1.02-2.69) and lower FEV1.

\section{Discussion}

In a cohort of COPD patients admitted to hospital with acute respiratory symptoms, the majority of patients with radiographic consolidation did not have pneumonia included in their discharge diagnoses. The presence of consolidation was associated with poorer outcomes and recurrent pneumonia was associated with use of ICSs.

\section{TABLE 4 Organisms isolated in sputum according to radiological diagnosis}

$\begin{array}{lcc}\text { Organism } & \text { Exacerbation } & \text { Pneumonia } \\ \text { Haemophilus influenzae } & 20(2.84 \%) & 10(4.26 \%) \\ \text { Streptococcus pneumoniae } & 8(1.14 \%) & 9(3.83) \\ \text { Moraxella catarrhalis } & 3(0.43 \%) & 0(0 \%) \\ \text { Pseudomonas aeruginosa } & 35(4.98 \%) & 17(7.23 \%) \\ \text { Klebsiella species } & 2(0.28 & 5(2.13 \%) \\ \text { Other Gram-negative bacteria } & 59(8.39 \%) & 23(9.79 \%) \\ \text { Escherichia coli } & 17(2.4 \%) & 9(3.83 \%) \\ \text { Coliform species } & 32(4.55 \%) & 11(4.68 \%) \\ \text { Enterobacter species } & 1(0.14 \%) & 2(0.85) \\ \text { Proteus species } & 2(0.28 \%) & 1(0.43 \%) \\ \text { Stenotrophomonas species } & 2(0.28 \%) & 0 \\ \text { Serratia species } & 2(0.28 \%) & 0 \\ \text { Staphylococcus aureus } & 1(0.14 \%) & 0 \\ \text { Total } & 15.0 \% & 28.0 \%\end{array}$


The relationship between COPD exacerbations and pneumonia is complex as pneumonia is sometimes considered to be a cause of COPD exacerbations [11-14] whereas others consider them separate clinical and pathological entities $[15,16]$. This lack of clarity is unsurprising as definitions of the relationship between pneumonia and COPD exacerbations in COPD guidelines are inconsistent. A 1995 European Respiratory Society (ERS) consensus statement included pneumonia as a cause of COPD exacerbation [17] and the joint American Thoracic Society/ERS 2004 COPD guidelines listed pneumonia as one of several high-risk comorbid conditions that may be present in patients with COPD exacerbations [18]. The 2013 GOLD strategy document included pneumonia in a list of conditions that can "mimic/aggravate COPD exacerbations" [19]; however, in the 2017 GOLD strategy, this was changed to state that "exacerbations must be differentiated clinically from other events such as... pneumonia" [8], inferring that exacerbations and pneumonia are separate, mutually exclusive events, an approach supported in a recent expert review [20]. In the older iterations of both the BTS and the National Institute for Health and Care Excellence (NICE) COPD guidelines, pneumonia was listed as among "other conditions that may present with similar symptoms in COPD patients and that must be excluded when making a diagnosis of an exacerbation" [21]. However, in the 2018 update of the NICE COPD guideline, this statement had been removed. The presence of "changes on chest radiograph" was listed as a factor favouring hospital admission, implying that consolidation does not exclude the diagnosis of COPD exacerbation as had been the case in previous versions of the guidelines [22].

Therefore, it is unsurprising that there is confusion as to what diagnostic label to use in hospitalised COPD patients with radiographic consolidation. This has resulted in the use of a number of different terms such as "pneumonic exacerbation of COPD", "COPD exacerbation with consolidation", "community-acquired pneumonia in a patient with COPD" and "COPD exacerbation and pneumonia", none of which has been universally accepted [11, 15,23]. Clinicians often make a diagnosis of acute exacerbation in COPD patients even when there is radiographic evidence of consolidation. The BTS and ERS COPD audits recruited patients with a primary diagnosis of COPD exacerbation but pneumonia was reported in up to $18 \%$ of patients $[9,11,24]$. Similar findings have also been reported in the USA [10]. However, there are enormous variations in the frequency of reported pneumonia in COPD patients. In the 13 individual countries included in the ERS audit, pneumonia rates ranged from $9.8 \%$ to 62.5\% [25], and this occurred even within the same country, with reported pneumonia ranging from $18.7 \%$ to $34.4 \%$ in six UK hospitals [12]. It is improbable that this variation is due to true geographical differences in the frequency of pneumonia. We believe it reflects the different opinions of clinicians as to whether the primary diagnosis of COPD patients who present with an acute respiratory illness and radiographic consolidation should be acute COPD exacerbation or pneumonia.

This variability in diagnostic labels makes it difficult to determine the true relationship between pneumonia and COPD exacerbations. In the BTS and ERS audits, chest radiograph interpretation and the decision to include or exclude patients depended on the individual clinicians' opinions and no information was available on pneumonia in excluded patients. Ours is the first study to analyse all COPD patients hospitalised with an acute respiratory illness irrespective of the treating clinicians' diagnoses, and to independently verify the presence of consolidation on the chest radiograph. In addition, all patients had spirometrically confirmed COPD whereas in the BTS and the ERS audits, only $46 \%$ and $51 \%$ of patients, respectively, had spirometry, and in the latter, $12.9 \%$ had spirometry incompatible with a diagnosis of COPD $[24,26]$.

We report that $16 \%$ of COPD patients had a discharge diagnosis of pneumonia, similar to the findings of the BTS and ERS audits [11, 24, 27]. However, radiographic consolidation was present in 25\% of admissions, suggesting that the true frequency of pneumonia in COPD patients is considerably higher. The discrepancy between this figure and the lower prevalence reported in the COPD audits is likely to reflect those patients excluded because pneumonia was considered the primary diagnosis. In $51.2 \%$ of patients with consolidation, pneumonia was not included as a discharge diagnosis, suggesting that clinicians are almost evenly split as to whether COPD patients with consolidation should be diagnosed with pneumonia.

In hospitalised COPD patients, a clinical diagnosis of pneumonia has been associated with greater length of stay and higher risk of intensive care unit admission and mortality [11, 24, 27-30]. In keeping with these studies, the presence of consolidation was associated with increased length of stay, higher rates of admission to a high-dependency unit and increased in-hospital mortality. This, together with higher inflammatory markers, suggest that the presence of consolidation is not just an incidental finding but has real clinical significance. Therefore, failure to correctly recognise and diagnose pneumonia in COPD patients will result in failure to identify high-risk patients.

Most patients $(97.56 \%)$ with a discharge diagnosis of COPD exacerbation were treated with corticosteroids. In patients with a discharge diagnosis of pneumonia, 56\% received corticosteroids, suggesting that 
clinicians are almost equally divided in their opinion as to whether to use corticosteroids in these patients. Oral corticosteroids do not reduce mortality in pneumonia [31] but improve lung function and reduce treatment failure rates in COPD exacerbations [32]. A retrospective analysis of corticosteroids suggested that they are not beneficial in COPD patients with pneumonia [33] although a randomised trial has not been carried out. Clinical trials in COPD exacerbations have excluded patients with pneumonia [34, 35] and therefore clinicians are unclear whether hospitalised COPD patients with consolidation should be treated according to pneumonia guidelines (no corticosteroids) or COPD guidelines (use corticosteroids). The use of a range of different antibiotics in our study also supports this assertion.

ICSs are commonly prescribed in COPD patients [36]; however, an association has been reported between ICSs and risk of pneumonia in COPD [3, 36, 37]. While there was no difference in overall ICS use between patients with and without consolidation in our cohort, ICS use was an independent risk factor for recurrent admissions with consolidation. This has been reported in a general hospitalised population [38] but this ours the first report specifically in COPD patients. A recent expert opinion recommended that ICS use should be avoided in COPD patients with repeated pneumonia events but used in patients with a history of hospitalisation for COPD exacerbation [39]. In 51.2\% of the admissions, the hospital discharge diagnosis was COPD exacerbation but radiographic consolidation was present. Therefore, relying on the recorded discharge diagnosis could lead to the incorrect use of ICSs in COPD patients.

Our study has several strengths and weaknesses. Its strengths are that all patients with a confirmed diagnosis of COPD admitted to the participating hospitals with an acute respiratory illness were included and the chest radiograph was independently assessed by two clinicians. The main weakness is that the study was limited to three UK hospitals and it is not known whether the results are specific to these institutions. However, the frequency of pneumonia as a discharge diagnosis was almost identical to that reported in the UK and European COPD audits, suggesting that our results are consistent with practice in other hospitals. The patients were admitted under clinicians from different specialties and we did not examine whether this had an impact on the interpretation of the chest radiograph and the diagnosis of pneumonia. Pneumonia was diagnosed on chest radiography but computed tomography (CT) scan has higher sensitivity for detecting pneumonia. However, CT scan is not routinely used in hospitalised patients with COPD exacerbations and therefore our study reflects real-life practice. Further studies using similar methodologies will confirm whether our findings are more widely applicable.

\section{Conclusions}

Our study suggests that pneumonia is frequently not recorded as a diagnosis in COPD patients admitted to hospital with an acute respiratory illness and radiographic consolidation. Consolidation was associated with worse clinical outcomes and, therefore, failure to diagnose pneumonia may hinder recognition of high-risk patients and contribute to incorrect use of ICSs in COPD patients. Further research is needed to understand the reasons for the underdiagnosis of pneumonia. We believe it is related to confusion regarding the correct diagnostic label to use in acutely unwell COPD patients with radiographic consolidation. This confusion stems from two different diagnostic approaches that can be taken in these patients; either to consider pneumonia as the primary diagnosis and COPD as a comorbidity or to consider COPD exacerbation as the primary diagnosis and pneumonia as a cause of the exacerbation. Current COPD guidelines offer no guidance on which is the correct approach. Future guidelines should specifically address the issue of pneumonia in COPD patients rather than simply stating that pneumonia should be excluded. A single terminology should be agreed upon so that further studies may be carried out investigating the prevalence, aetiology, risk factors and outcomes of pneumonia in COPD, and provide a basis for clinical trials investigating treatments. These studies will be essential for the development of evidence-based guidelines to improve management of COPD patients with pneumonia.

Conflict of interest: None declared.

\section{References}

1 Mathers CD, Loncar D. Projections of global mortality and burden of disease from 2002 to 2030. PLoS Med 2006; 3: e442.

2 Farr BM, Woodhead MA, Macfarlane JT, et al. Risk factors for community-acquired pneumonia diagnosed by general practitioners in the community. Respir Med 2000; 94: 422-427.

3 Janson C, Johansson G, Stallberg B, et al. Identifying the associated risks of pneumonia in COPD patients: ARCTIC an observational study. Respir Res 2018; 19: 172.

4 National Institute for Health and Care Excellence. Pneumonia in adults: diagnosis and management. https://www. nice.org.uk/guidance/cg191. Date last updated: December 3, 2014.

5 van Vugt SF, Verheij TJ, de Jong PA, et al. Diagnosing pneumonia in patients with acute cough: clinical judgment compared to chest radiography. Eur Respir J 2013; 42: 1076-1082.

6 Elemraid MA, Muller M, Spencer DA, et al. Accuracy of the interpretation of chest radiographs for the diagnosis of paediatric pneumonia. PLoS One 2014; 9: e106051. 
7 Lieberman D, Shvartzman P, Korsonsky I, et al. Diagnosis of ambulatory community-acquired pneumonia Comparison of clinical assessment versus chest X-ray. Scand J Prim Health Care 2003; 21: 57-60.

8 Vogelmeier CF, Criner GJ, Martinez FJ, et al. Global Strategy for the Diagnosis, Management, and Prevention of Chronic Obstructive Lung Disease 2017 Report: GOLD Executive Summary. Am J Respir Crit Care Med 2017; 195: 557-582.

9 Saleh A, Lopez-Campos JL, Hartl S, et al. The effect of incidental consolidation on management and outcomes in COPD exacerbations: data from the European COPD Audit. PLoS One 2015; 10: e0134004.

10 Bustamante-Fermosel A, De Miguel-Yanes JM, Duffort-Falco M, et al. Mortality-related factors after hospitalization for acute exacerbation of chronic obstructive pulmonary disease: the burden of clinical features. Am J Emerg Med 2007; 25: 515-522.

11 Myint PK, Lowe D, Stone RA, et al. U.K. National COPD Resources and Outcomes Project 2008: patients with chronic obstructive pulmonary disease exacerbations who present with radiological pneumonia have worse outcome compared to those with non-pneumonic chronic obstructive pulmonary disease exacerbations. Respiration 2011; 82: 320-327.

12 Echevarria C, Steer J, Heslop-Marshall $\mathrm{K}$, et al. Validation of the DECAF score to predict hospital mortality in acute exacerbations of COPD. Thorax 2016; 71: 133-140.

13 Steer J, Gibson J, Bourke SC. The DECAF Score: predicting hospital mortality in exacerbations of chronic obstructive pulmonary disease. Thorax 2012; 67: 970-976.

14 Roche N, Zureik M, Soussan D, et al. Predictors of outcomes in COPD exacerbation cases presenting to the emergency department. Eur Respir J 2008; 32: 953-961.

15 Crisafulli E, Menendez R, Huerta A, et al. Systemic inflammatory pattern of patients with community-acquired pneumonia with and without COPD. Chest 2013; 143: 1009-1017.

16 Huerta A, Crisafulli E, Menendez R. Pneumonic and nonpneumonic exacerbations of COPD: inflammatory response and clinical characteristics. Chest 2013; 144: 1134-1142.

17 Siafakas NM, Vermeire P, Pride NB, et al. Optimal assessment and management of chronic obstructive pulmonary disease (COPD). Eur Respir J 1995; 8: 1398-1420.

18 Celli BR, MacNee W, ATS/ERS Task Force. Standards for the diagnosis and treatment of patients with COPD: a summary of the ATS/ERS position paper. Eur Respir J 2004; 23: 932-946.

19 Vestbo J, Hurd SS, Agusti AG, et al. Global strategy for the diagnosis, management, and prevention of chronic obstructive pulmonary disease: GOLD executive summary. Am J Respir Crit Care Med 2013; 187: 347-365.

20 Kim V, Aaron SD. What is a COPD exacerbation? Current definitions, pitfalls, challenges and opportunities for improvement. Eur Respir J 2018; 52: 1801261.

21 The COPD Guidelines Group of the Standards of Care Committee of the BTS. BTS guidelines for the management of chronic obstructive pulmonary disease. Thorax 1997; 52: Suppl. 5, S1-S28.

22 National Institute for Health and Care Excellence. Chronic obstructive pulmonary disease in over 16s: diagnosis and management. www.nice.org.uk/guidance/ng115. Date last updated: December 2018.

23 Hurst JR. Consolidation and exacerbation of COPD. Med Sci 2018; 6: E44.

24 Royal College of Physicians. COPD: Who Cares When It Matters Most? www.rcplondon.ac.uk/projects/outputs/ copd-who-cares-when-it-matters-most-outcomes-report-2014 Date last updated: February 1, 2017.

25 Roberts CM, Hartl S, López-Campos JL. An International Comparison of COPD Care in Europe: Results of the First European COPD Audit. Sheffield, European Respiratory Society, 2012.

26 Lopez-Campos JL, Castro-Acosta A, Pozo-Rodriguez F, et al. Accuracy of COPD diagnosis during an admission the european perspective. Chest 2017; 151: 1396-1397.

27 Roberts CM, Lopez-Campos JL, Pozo-Rodriguez F, et al. European hospital adherence to GOLD recommendations for chronic obstructive pulmonary disease (COPD) exacerbation admissions. Thorax 2013; 68: 1169-1171.

28 Chen D, Restrepo MI, Fine MJ, et al. Observational study of inhaled corticosteroids on outcomes for COPD patients with pneumonia. Am J Respir Crit Care Med 2011; 184: 312-316.

29 Sogaard M, Madsen M, Lokke A, et al. Incidence and outcomes of patients hospitalized with COPD exacerbation with and without pneumonia. Int J COPD 2016; 11: 455-465.

30 Sharafkhaneh A, Tavakoli-Tabasi S, Musher D. Mortality in patients admitted for concurrent COPD exacerbation and pneumonia. COPD 2017; 14: 462.

31 Wan YD, Sun TW, Liu ZQ, et al. Efficacy and safety of corticosteroids for community-acquired pneumonia: a systematic review and meta-analysis. Chest 2016; 149: 209-219.

32 Walters JA, Tan DJ, White CJ, et al. Systemic corticosteroids for acute exacerbations of chronic obstructive pulmonary disease. Cochrane Database Syst Rev 2014; 9: CD001288.

33 Scholl T, Kiser TH, Vondracek SF. Evaluation of systemic corticosteroids in patients with an acute exacerbation of COPD and a diagnosis of pneumonia. Chronic Obstr Pulm Dis 2018; 5: 57-65.

34 Leuppi JD, Schuetz P, Bingisser R, et al. Short-term versus conventional glucocorticoid therapy in acute exacerbations of chronic obstructive pulmonary disease: the REDUCE randomized clinical trial. JAMA 2013; 309: 2223-2231.

35 Llor C, Moragas A, Hernandez S, et al. Efficacy of antibiotic therapy for acute exacerbations of mild to moderate chronic obstructive pulmonary disease. Am J Respir Crit Care Med 2012; 186: 716-723.

36 Lipson DA, Barnhart F, Brealey N, et al. Once-daily single-inhaler triple versus dual therapy in patients with COPD. N Engl J Med 2018; 378: 1671-1680.

37 Finney L, Berry M, Singanayagam A, et al. Inhaled corticosteroids and pneumonia in chronic obstructive pulmonary disease. Lancet Respir Med 2014; 2: 919-932.

38 Eurich DT, Lee C, Marrie TJ, et al. Inhaled corticosteroids and risk of recurrent pneumonia: a population-based, nested case-control study. Clin Infect Dis 2013; 57: 1138-1144

39 Agusti A, Fabbri LM, Singh D, et al. Inhaled corticosteroids in COPD: Friend or foe? Eur Respir J 2018; 52 1801219. 